\title{
EFFECT OF HEAT TRANSFER ON PERISTALTIC FLOW IN PRESENCE OF HEAT GENERATION AGAINST HIGHER VALUE OF REYNOLDS NUMBER USING FEM
}

\author{
TARIQ JAVED
}

Department of Mathematics and Statistics, International Islamic University Islamabad, Pakistan

ABdul H. HAmid

Department of Mathematics, Division of Science and Technology, University of Education, Lahore, Pakistan

e-mail: ahhamid51@gmail.com

Bilal Ahmed

Department of Mathematics and Statistics, The University of Lahore, Pakistan

NASIR Ali

Department of Mathematics and Statistics, International Islamic University Islamabad, Pakistan

\begin{abstract}
In the present investigation, heat transfer analysis of peristaltic flow in the presence of heat generation against high values of the Reynolds number using a numerical technique is presented. The Finite Element Method with the assistance of Galerkin's variational method has been used on the governing Navier-Stokes equation in the stream-vorticity form. The graphs of computed longitudinal velocity, temperature distribution and pressure rise per wavelength are plotted against different values of an emerging parameter using a stream function and vorticity. The presented results are valid beyond the restrictions of long wavelength and low Reynolds number limits. Moreover, large values of the Reynolds number increases pressure rise per wavelength.
\end{abstract}

Keywords: finite element method, peristaltic flow, heat transfer

\section{Introduction}

The phenomena of wave relaxation and contraction incorporated by the walls of a flexible tube are known as the peristaltic fluid. These flows have a key role in fluid transportation for both the living organism and industrial pumping. Blood flow through arteries and in small blood vessels, gastrointestinal tract, transportation of urine from the kidney to the urinary bladder, bile duct/glandular ducts, etc. are examples of physiology flows. This principle is also found in many biomedical instruments like heart-lung machine and blood pumps used in dialysis. The peristaltic motion also occurs in many industrial applications like transportation of the toxic liquid used in the nuclear industry which is used to avoid contamination from the outside environment and also in sanitary fluid transportation in many other situations. In the treatment of diseased tissues, the heat transfer is also significantly used in the peristaltic motion. The theoretical analysis of two-dimensional peristaltic flows for finite Reynolds numbers (Re) is challenging due to nonlinearity amid the flow field and moving wall. Most of the analytical studies used perturbation series against the small parameter and, in consequence, the validation range is small there. However, this technique does offer categorical information about the physical effects of the parameters, and the obtained results are helpful for checking the accuracy of the numerical methods.

After the innovatory works of Latham (1966) in his master thesis, Shapiro et al. (1969) and Fung and Yih (1969) on peristaltic flow, we found that many analytical, several investigations 
have been carried out theoretically or experimentally (Weinberg et al., 1971; Lew et al., 1971; Jaffrin, 1973; Takabatake and Ayukawa, 1982, Takabatake et al., 1987, 1988, 1990; Kumar and Naidu, 1995; Hayat et al., 2005). The common thing in these studies is that they all considered long wavelength and low Reynolds number assumptions because these type of studies are extremely difficult in the presence of higher order non-linearity between the flow field and the moving wall. Recent investigations (Ali et al., 2008; Hayat et al., 2008; Mekheimer, 2008; Tripathi, 2013) dealing with nonlinear two-dimensional peristaltic flows give the analytical solution by considering the same assumptions discussed earlier. In numerical analysis, Takabatake et al. (1988) employed the finite difference method to solve the Navier-Stokes equations for two-dimensional peristaltic flow problems for moderate Reynolds numbers and wave numbers. Takabatake et al. $(1987,1990)$ also employed finite element methods for two-dimensional peristaltic flows. Kumar and Naidu (1995) also applied the finite element method and obtained results which were convergent up to Re $=100$. Hayat et al. (2005) considered the Johnson-Segalman fluid transported peristaltically under the inducement of a magnetic field.

Yi et al. (2002) carried out the study of flow between two vibrating peristaltic walls in a closed cavity. The heat and mass transfer effects received considerable attention from many researchers because of their several applications in the chemical industry. The slip effects and heat transfer on peristaltic flow were carried out by Hayat et al. (2010). Kothandapani and Srinivas (2008) discussed the peristaltic flow through a porous space with compliant walls. They observed the effects of heat and mass transfer with magnetic effects and observed that the heat effects were less in a curved channel in comparison to a straight channel. Many more studies were also found on heat transfer in literature (Srinivas and Kothandapani, 2008; Eldabe et al., 2008; El-Sayed et al., 2011; Abo-Eldahab et al., 2011).

In this article, we discussed the numerical solution for nonlinear heat transfer on peristaltic flow using the finite element method. The non-linear governing PDE are reduced to the stream function-vorticity $\psi-\omega$. The stream-vorticity form has advantages over using a simple form of Navier-Stokes equation. The main advantage of using the stream-vorticity $\psi$ - $\omega$ formulation is that it helps us to reduce the number of unknowns from three to two. The other advantage using this formulation is that the vorticity is computed directly from the governing equations and does not find the curl of the velocity field. Also, it requires no restriction on the magnitudes of different parameters involved in the equation. The solution obtained by this formalism is utilized to illustrate the effects of moderate Reynolds numbers and wave numbers on various features of peristaltic motion. The results obtained through the present scheme are also compared with the existing results.

\section{Problem formulation}

Here, consider two-dimensional unsteady, incompressible viscous flow in an infinite channel. The width of the channel is $2 a$. The upper wall of peristaltic flow is maintained at temperature $T_{1}$. Assume that the walls of the channel are electrically insulated and move peristaltically with velocity $c$. The Cartesian coordinate system $(X, Y)$ is used in such a way that propagation of the wave is along the $X$-axis, and the $Y$-axis is considered perpendicular to the direction of the flow (see Fig. 1).

The $H(X, t)$ is the domain of the problem and is defined as

$$
H(X, t)=a-b \cos \frac{2 \pi(X-c t)}{\lambda}
$$

where $\lambda, a$ and $b$ are the wave length, mean distance of the wall from the central axis and the wave amplitude respectively. $c$ is the velocity with which the sinusoidal wave progresses along 


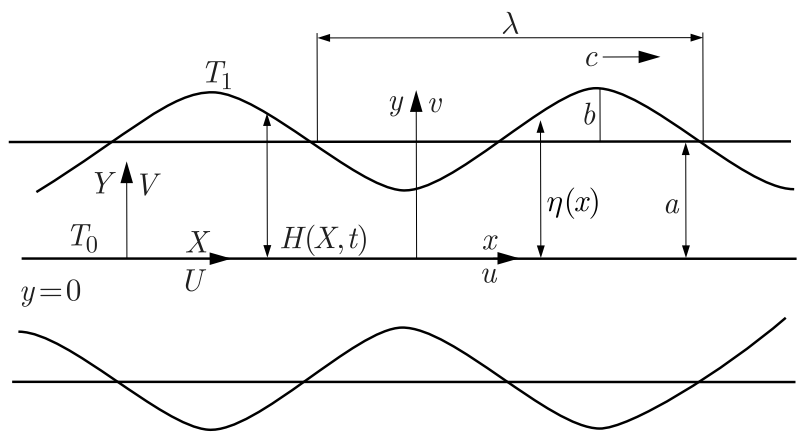

Fig. 1. Geometry of the flow problem

the wall in the $X$-direction. Using the velocity relations $x^{*}=X-c t, y^{*}=Y, u^{*}=U-c$ and $v^{*}=V$ from the lab frame to the wave frame, where $U, V$ and $u, v$ are the corrdinates of velocity in the lab and wave frames, respectively. The governing equations in the wave frame of reference are as follows

$$
\frac{\partial u^{*}}{\partial x^{*}}+\frac{\partial v^{*}}{\partial y^{*}}=0
$$

and

$$
\begin{aligned}
& \rho\left(u^{*} \frac{\partial u^{*}}{\partial x^{*}}+v^{*} \frac{\partial u^{*}}{\partial y^{*}}\right)=-\frac{\partial p^{*}}{\partial x^{*}}+\mu\left(\frac{\partial^{2} u^{*}}{\partial x^{* 2}}+\frac{\partial^{2} u^{*}}{\partial y^{* 2}}\right) \\
& \rho\left(u^{*} \frac{\partial v^{*}}{\partial x^{*}}+v^{*} \frac{\partial v^{*}}{\partial y^{*}}\right)=-\frac{\partial p^{*}}{\partial y^{*}}+\mu\left(\frac{\partial^{2} v^{*}}{\partial x^{* 2}}+\frac{\partial^{2} v^{*}}{\partial y^{* 2}}\right) \\
& \rho c_{p}\left(u^{*} \frac{\partial T^{*}}{\partial x^{*}}+v^{*} \frac{\partial T^{*}}{\partial y^{*}}\right)=\kappa^{*}\left(\frac{\partial^{2} T^{*}}{\partial x^{* 2}}+\frac{\partial^{2} T^{*}}{\partial y^{* 2}}\right)+Q_{0}
\end{aligned}
$$

where $Q_{0}$ is the heat generation parameter. In the wave frame, equation (2.1) takes the form

$$
\eta\left(x^{*}\right)=a-b \cos \left(\frac{2 \pi x^{*}}{\lambda^{*}}\right)
$$

and the boundary conditions are as follows:

- at $y^{*}=0$

$$
v^{*}=0 \quad \frac{\partial u^{*}}{\partial y^{*}}=0 \quad \frac{\partial T^{*}}{\partial y^{*}}=0
$$

— at $y^{*}=\eta\left(x^{*}\right)$

$$
u^{*}=c \quad v^{*}=\frac{2 \pi b}{\lambda} \sin \frac{2 \pi x^{*}}{\lambda} \quad T^{*}=T_{1}
$$

Since the flow rate $q^{*}$ in the wave frame is constant at each cross section of the channel, so we obtained an extra boundary condition, i.e.

$$
\psi^{*}=0 \quad \text { at } \quad y^{*}=0 \quad \text { and } \quad \psi^{*}=q^{*} \quad \text { at } \quad y^{*}=\eta\left(x^{*}\right)
$$

The flow rate in the wave frame $q^{*}$ and the time mean flow in the laboratory frame $Q^{*}$ can be related with the expression $q^{*}=Q^{*}-c H$. The following dimensionless variables are introduced to make the problem simple and general

$$
\begin{aligned}
& u=\frac{u^{*}}{c} \quad v=\frac{v^{*}}{c} \quad x=\frac{x^{*}}{\lambda} \\
& y=\frac{y^{*}}{a} \quad \psi=\frac{\psi^{*}}{c a} \quad q=\frac{q^{*}}{c h} \\
& \eta=\frac{\eta^{*}}{a} \quad p=\frac{a^{2}}{\lambda \mu c} p^{*}\left(x^{*}\right) \quad \theta=\frac{T^{*}-T_{0}}{T_{1}-T_{0}} \quad \beta=\frac{Q_{0} a^{2}}{\kappa^{*}\left(T_{1}-T_{0}\right)}
\end{aligned}
$$


where $T_{1}$ and $T_{0}$ are the surface and initial temperatures, respectively. Using the velocity stream function relation $u=\partial \psi / \partial y$ and $v=-\alpha \partial \psi / \partial x$ in Eqs. (2.3) and (2.4) and eliminating the pressure gradient, we have the following system of equations in the stream-vorticity form

$$
\alpha^{2} \frac{\partial^{2} \psi}{\partial x^{2}}+\frac{\partial^{2} \psi}{\partial y^{2}}=-\omega
$$

and

$$
\operatorname{Re}\left(\frac{\partial \psi}{\partial y} \frac{\partial \omega}{\partial x}-\frac{\partial \psi}{\partial x} \frac{\partial \omega}{\partial y}\right)=\nabla^{2} \omega \quad \operatorname{Re} \operatorname{Pr}\left(\frac{\partial \psi}{\partial y} \frac{\partial \theta}{\partial x}-\frac{\partial \psi}{\partial x} \frac{\partial \theta}{\partial y}\right)=\nabla^{2} \theta+\beta
$$

where $\operatorname{Re}=c a \alpha / \nu, \operatorname{Pr}=\mu c_{p} / \kappa^{\prime}, \alpha=a / \lambda$ and $\beta$ are the Reynolds number, Prandtl number, wave number and heat generation parameter, respectively. The boundary conditions in terms of $\psi(x, y)$ are reduced to:

- at $y=0$

$$
\psi=0 \quad \frac{\partial^{2} \psi}{\partial y^{2}}=0 \quad \frac{\partial \psi}{\partial x}=0 \quad \frac{\partial \theta}{\partial y}=0
$$

- at $y=\eta(x)$

$$
\psi=q \quad \frac{\partial \psi}{\partial y}=-1 \quad \frac{\partial \psi}{\partial x}=2 \pi \phi \sin (2 \pi x) \quad \theta=1
$$

in which

$$
\omega=\alpha \frac{\partial v}{\partial x}-\frac{\partial u}{\partial y} \quad \nabla^{2}=\alpha^{2} \frac{\partial^{2}}{\partial x^{2}}+\frac{\partial^{2}}{\partial y^{2}}
$$

\section{Numerical analysis}

For the numerical analysis of the current model, governing equations (2.8) and (2.9) with boundary conditions (2.10) and (2.11) are numerically solved by using the finite element method. It is important to note that when $\mathbf{R e} \rightarrow 0$ and $\alpha \rightarrow 0$, Eqs. (2.9) reduce to the ordinary differential equation. Due to continuity of the flow, we consider one wave at a time and then move to the second one and so on. In all the cases, highly convergent results have been obtained in 2-4 iterations with non-uniform meshing using pdetool in MATLAB for different values of the parameters involved. The stream function and vorticity is approximated by

$$
\psi=\sum_{k=1}^{n} \psi_{k} N_{k} \quad \theta=\sum_{k=1}^{n} \theta_{k} N_{k} \quad \omega=\sum_{k=1}^{n} \omega_{k} N_{k}
$$

where $\psi_{k}$ and $\omega_{k}$ are the element nodal approximations of $\psi$ and $\omega$. Then the Galerkin finite element method is applied to governing equation $(3.2)_{2,3}$ as

$$
\begin{aligned}
& \int_{\Omega} w_{1}\left(\alpha^{2} \frac{\partial^{2} \psi}{\partial x^{2}}+\frac{\partial^{2} \psi}{\partial y^{2}}+\omega\right) d \Omega=0 \\
& \int_{\Omega} w_{2}\left[\operatorname{Re}\left(\frac{\partial \psi}{\partial y} \frac{\partial \omega}{\partial x}-\frac{\partial \psi}{\partial x} \frac{\partial \omega}{\partial y}\right)-\left(\alpha^{2} \frac{\partial^{2} \omega}{\partial x^{2}}+\frac{\partial^{2} \omega}{\partial y^{2}}\right)\right] d \Omega=0 \\
& \int_{\Omega} w_{3}\left[\operatorname{Re} \operatorname{Pr}\left(\frac{\partial \psi}{\partial y} \frac{\partial \theta}{\partial x}-\frac{\partial \psi}{\partial x} \frac{\partial \theta}{\partial y}\right)-\left(\alpha^{2} \frac{\partial^{2} \theta}{\partial x^{2}}+\frac{\partial^{2} \theta}{\partial y^{2}}\right)-\beta\right] d \Omega=0
\end{aligned}
$$


where $w_{1}, w_{2}$ and $w_{3}$ are weight functions. After simplifying equation (3.2), we obtain

$$
\begin{aligned}
& \int_{\Omega}\left(\alpha^{2} \frac{\partial w_{1}}{\partial x} \frac{\partial \psi}{\partial x}+\frac{\partial w_{1}}{\partial y} \frac{\partial \psi}{\partial y}\right) d \Omega-\int_{\Omega} w_{1} \omega d \Omega=\int_{\Gamma} w_{1} \frac{\partial \psi}{\partial n} d \Gamma \\
& \int_{\Omega} \operatorname{Re} w_{2}\left(\frac{\partial \psi}{\partial y} \frac{\partial \omega}{\partial x}-\frac{\partial \psi}{\partial x} \frac{\partial \omega}{\partial y}\right) d \Omega-\int_{\Omega}\left(\alpha^{2} \frac{\partial w_{2}}{\partial x} \frac{\partial \omega}{\partial x}+\frac{\partial w_{2}}{\partial y} \frac{\partial \omega}{\partial y}\right) d \Omega=\int_{\Gamma} w_{2} \frac{\partial \omega}{\partial n} d \Gamma \\
& \int_{\Omega} \operatorname{Re} \operatorname{Pr} w_{3}\left(\frac{\partial \psi}{\partial y} \frac{\partial \theta}{\partial x}-\frac{\partial \psi}{\partial x} \frac{\partial \theta}{\partial y}\right) d \Omega-\int_{\Omega} w_{3}\left(\alpha^{2} \frac{\partial \psi}{\partial y} \frac{\partial \theta}{\partial x}-\frac{\partial \psi}{\partial x} \frac{\partial \theta}{\partial y}\right) d \Omega-\int_{\Omega} w_{3} \beta d \Omega \\
& \quad=\int_{\Gamma} w_{3} \frac{\partial \theta}{\partial n} d \Gamma
\end{aligned}
$$

Introducing equation (3.1) into equations (3.3) and considering the discretized domain, we have

$$
\begin{aligned}
& -\sum_{i} B_{k i}^{e} \omega_{i}+\sum_{i} A_{k i}^{e} \psi_{i}=S_{n}^{k^{e}} \\
& \sum_{i} A_{k i}^{e} \omega_{i}+\operatorname{Re} \sum_{i, j} C_{k i j}^{e} \psi_{i} \omega_{i}=0 \\
& \sum_{i} A_{k i}^{e} \theta_{i}+\operatorname{Re} \operatorname{Pr} \sum_{i, j} C_{k i j}^{e} \psi_{i} \theta_{i}=S_{n}^{k^{e}}+\beta S^{k^{e}}
\end{aligned}
$$

where

$$
\begin{array}{rlrl}
A_{k i}^{e} & =\int_{\Omega^{e}}\left(\alpha^{2} \frac{\partial N_{k}}{\partial x} \frac{\partial N_{i}}{\partial x}+\frac{\partial N_{k}}{\partial y} \frac{\partial N_{i}}{\partial y}\right) d \Omega & B_{k i}^{e}=\int_{\Omega^{e}} N_{k} N_{i} d \Omega \\
C_{k i j}^{e}=\int_{\Omega^{e}} P_{k}\left(\frac{\partial N_{i}}{\partial y} \frac{\partial N_{j}}{\partial x}-\frac{\partial N_{j}}{\partial x} \frac{\partial N_{i}}{\partial y}\right) d \Omega^{e} & S^{k^{e}}=\int_{\Gamma} N_{k} d \Gamma \\
S_{n}^{k^{e}}=\int_{\Gamma} N_{k} \bar{S}_{k} d \Gamma &
\end{array}
$$

The global system in the matrix form is

$$
\mathbf{K A}=\mathbf{F}
$$

where the entries of the above matrices are

$$
\begin{aligned}
& \mathbf{K}=\left\{K_{i j}\right\}=\left[\begin{array}{ccc}
-B_{k i}^{e} & A_{k i}^{e} & 0 \\
A_{k i}^{e} & \operatorname{Re} C_{k i j}^{e} \omega_{i} & 0 \\
0 & \operatorname{Re} \operatorname{Pr} C_{k i j}^{e} \psi_{i} & -A_{k i}^{e}
\end{array}\right] \\
& \mathbf{A}=\left\{A_{k}\right\}=\left[\begin{array}{c}
\omega_{k} \\
\psi_{k} \\
\theta_{k}
\end{array}\right] \\
& \mathbf{F}=\left\{F_{k}\right\}=\left[\begin{array}{c}
S_{n}^{k^{e}} \\
0 \\
S_{n}^{k^{e}}+\beta S^{k^{e}}
\end{array}\right]
\end{aligned}
$$

The non-linear equations are then solved using the Newton-Raphson method. The solution process is iterated until the subsequent convergence condition is satisfied.

\section{Pressure analysis}

The pressure rise is an important part in the study of the peristaltic flow. To evaluate pressure in the channel, it is enough to evaluate only the central part of the wave. The pressure rise per wave length can be evaluated by the expression 


$$
\Delta P_{\lambda}=\int_{0}^{\lambda} \frac{\partial p}{\partial x} d x
$$

where $\partial p / \partial x$ is obtained directly from dimensionless form of the Navier-Stokes equation.

\section{Numerical results and discussion}

We discretize the considered domain into a mesh of triangular elements each containing six nodes per element. First, a solution on each triangular mesh is found and then they are assembled to a global system. Finally, the solution has been obtained by applying the Newton-Raphson method. In all cases, highly convergent results are obtained by our own code built in MATLAB with a tolerance of $10^{-14}$ in 3-5 iterations. Computation of the developed problem is performed in terms of velocity, streamlines in the wave and lab frames, vorticity and pressure against pertinent parameters including the amplitude ratio $\phi$, Reynold's number Re, volume flow rate $Q$, Prandtl number Pr, the wave number $\alpha$ and heat generation parameter $\beta$.

\subsection{Validation}

The results obtained by the developed code are validated with the result presented by Jaffrin (1973) as the limiting case, i.e. Re $\rightarrow 0$ and $\alpha \rightarrow 0$. The results presented by Jaffrin (1973) are valid for small values of the Reynolds and wave number. Figure 2 shows the computed pressure rise per wavelength for different values of $\phi$, when $\alpha=0.05$ and $\operatorname{Re}=1.0$ and results with Jaffrin (1973), which validates the accuracy of our computed results. Figure 3 presents the graph of the computed pressure rise for different values of the wave number with a fixed Reynolds number and the comparison with the result of Jaffrin (1973) and found in good agreement.

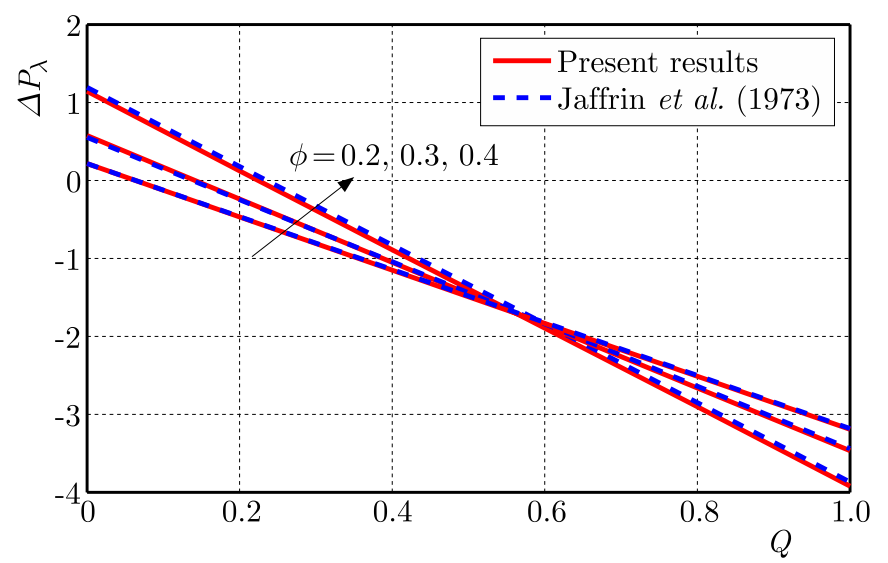

Fig. 2. Comparison of the computed pressure rise (solid line) with Jaffrin (1973) (dashed line) for $\alpha=0.05$ and $\operatorname{Re}=1.0$

\subsection{Velocity field}

In this Section, we discuss the behavior of longitudinal velocity $u$ and the temperature profile $\theta$ at $x=0$ for different values of the time mean flow $Q$, Reynolds number Re, the wave number $\alpha$, Prandtl number Pr and the heat generation parameter $\beta$ in Figs. 4-7. It is perceived that the velocity of the fluid has a cosine profile in the entire set of figures. In Fig. 4, we see that the longitudinal velocity increases in the whole region of the channel by increasing the values of the time mean flow $Q$, while an increase in the temperature profile is noted to be very 


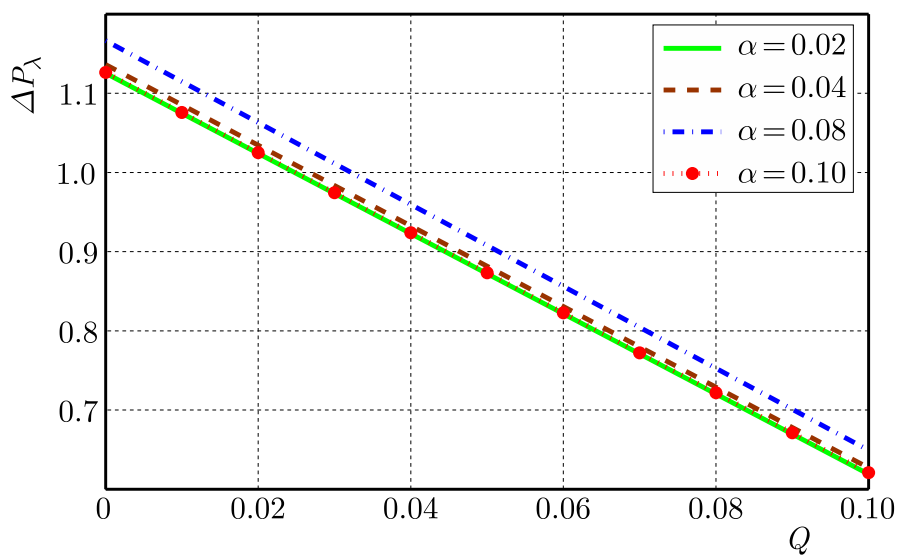

Fig. 3. Comparison of the computed pressure with for Jaffrin (1973) (dots) for different values of $\alpha$ with the fixed value $\operatorname{Re}=1.0$ and $\phi=0.5$

(a)

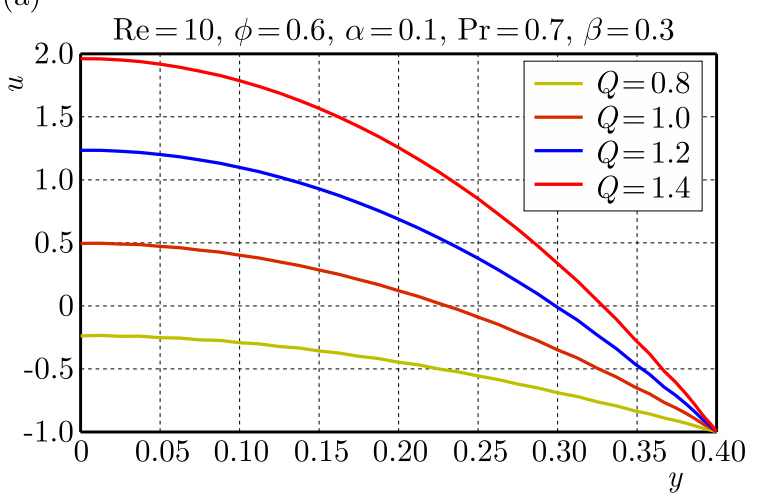

(b)

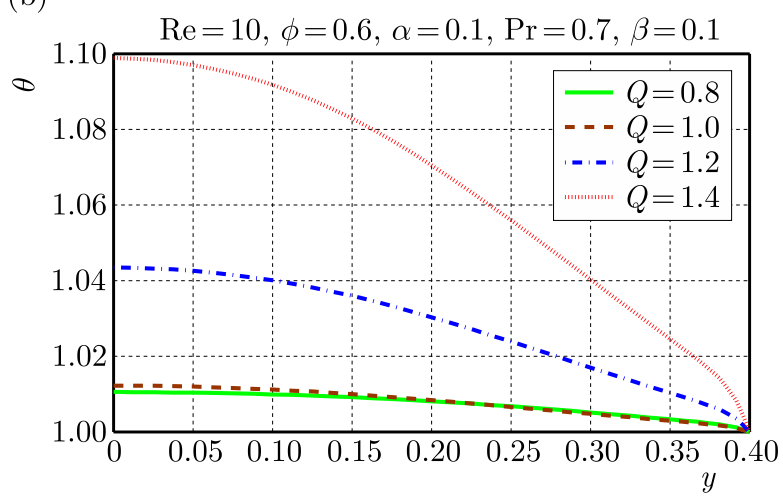

Fig. 4. (a) Longitudinal velocity and (b) temperature profile against different values of $Q$

(a)

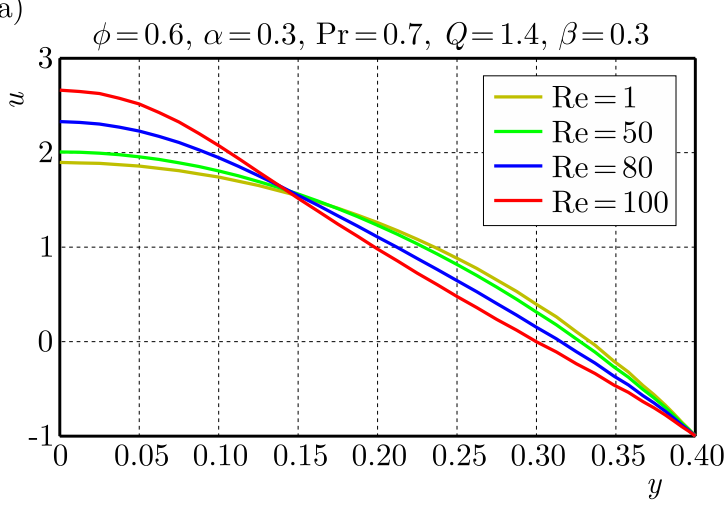

(b)

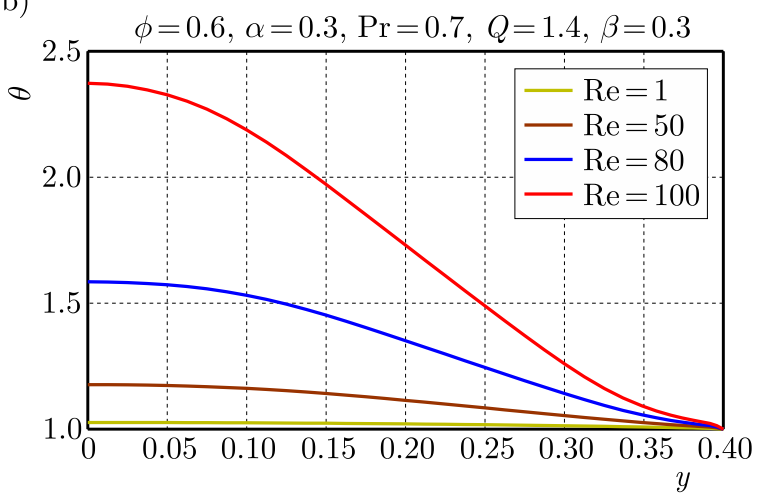

Fig. 5. (a) Longitudinal velocity and (b) temperature profile against different values of Re

rapid with the time mean flow $Q$. In Fig. 5a, the longitudinal velocity increases nearly up to the center of the channel with increasing values of the Reynolds number. However, increasing the wave number causes a decrease in the velocity up to the lower half of the channel and after it, the opposite behavior is observed as shown in Fig. 6a. Similarly, by increasing the value of the Reynolds number, the temperature profile shows increasing behavior. On the other hand, the wave number helps to control the temperature inside the channel as shown in Fig. 6b. In Figs. 7 and 8 , the temperature profile exhibits the same behavior of increasing nature with an increase in the values of $\beta$ and $\operatorname{Pr}$. 
(a)

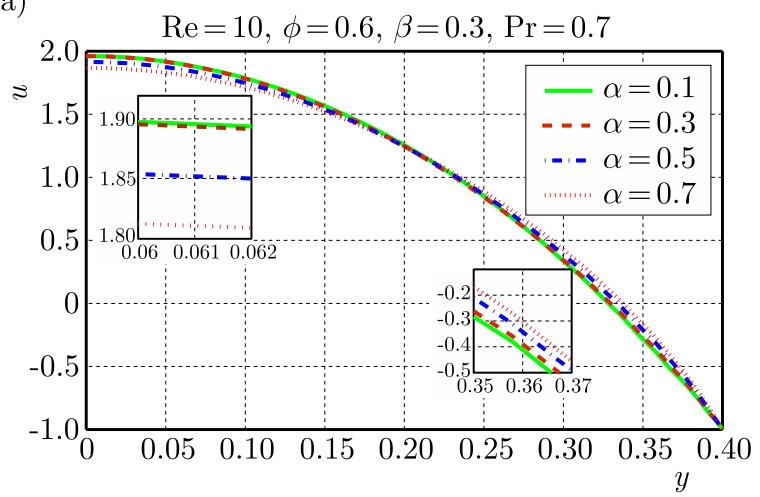

(b)

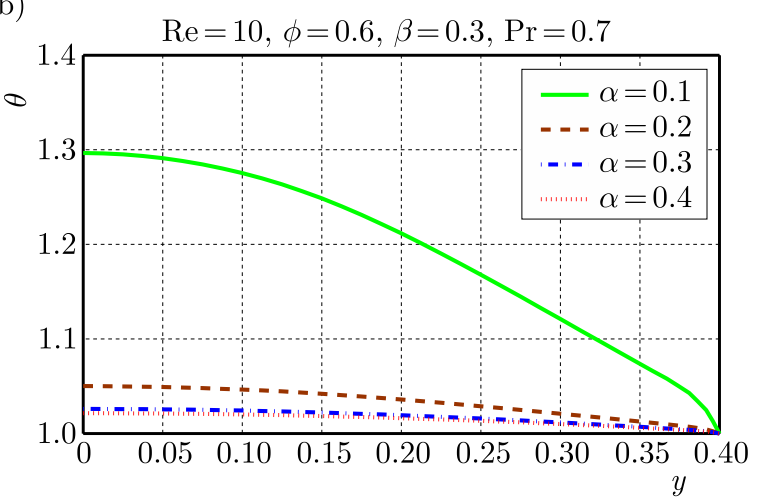

Fig. 6. (a) Longitudinal velocity and (b) temperature profile against different values of $\alpha$

(a)

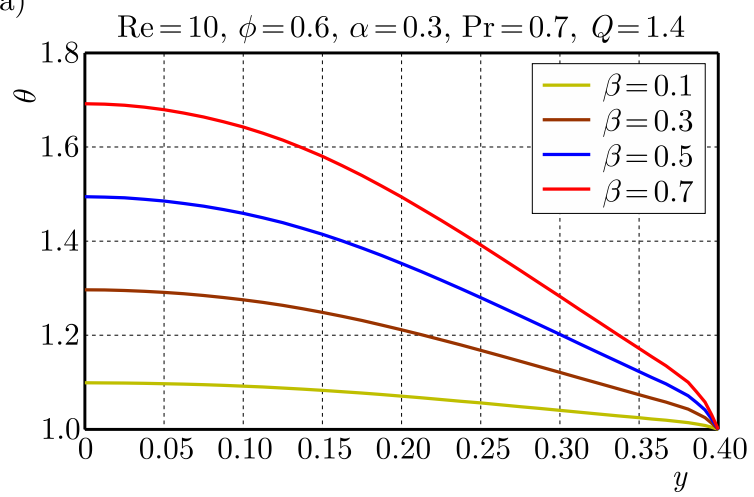

(b)

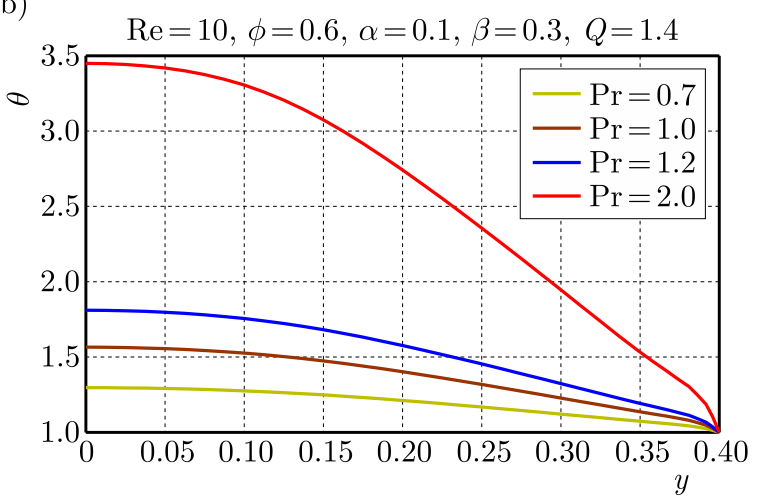

Fig. 7. Temperature profile for different values: (a) of $\beta$, (b) of $\operatorname{Pr}$

\subsection{Trapping}

In this Section, we discuss the most important feature of the peristaltic flow known as the trapping caused by splitting of streamlines and enclosing in circulating bolus of the fluid which behaves as a whole peristaltic wave. In Figs. 8-13, we observe the behavior of streamlines and isothermal lines for different values of the parameters in the flow domain. In Fig. 8, we observe that the trapping generously depends on the time-mean flow $Q$, i.e. the number of bolus decreases with increasing the magnitude of the time-mean flow and growing tendency to move towards the wall of the channel. In Fig. 9, we observe that an increase in the Reynolds number up to 100 causes a small increase in the size of the bolus. Figure 10 shows the behavior of isothermal lines for different values of the time mean flow which exhibits that an increase in this parameter causes an increase in the number of isothermal lines near the center of the channel towards the right side. On the other hand, the curvature effects on the isothermal lines near the center of the channel are reduced. In Fig. 11, we observe that by increasing the Reynolds number, the isothermal lines become smooth near the wall in the case of small values of the Reynolds number. The curvature effect increases near the wall of the channel as shown in Fig. 12 for increasing values of $\beta$. It is also noted that the number of isothermal lines increases by increasing the values of $\beta$. In Fig. 13, we notice that by increasing the value of $\mathrm{Pr}$, the curvature effect sharply increases and the number of isothermal lines also increases. 

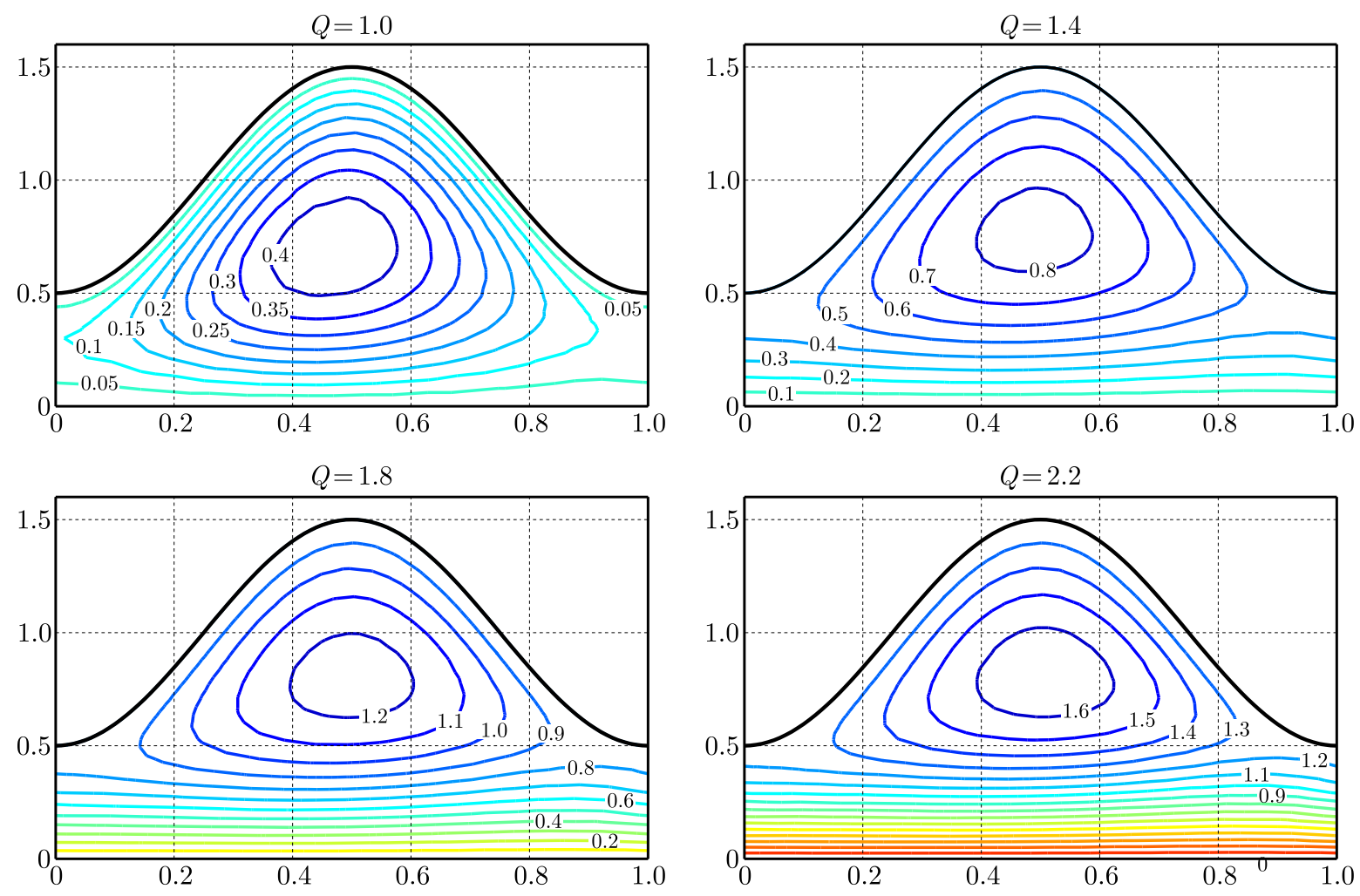

Fig. 8. Variation of stream lines in the wave frame for different values of $Q$ with $\operatorname{Re}=10$, $\phi=0.5, \alpha=0.5$
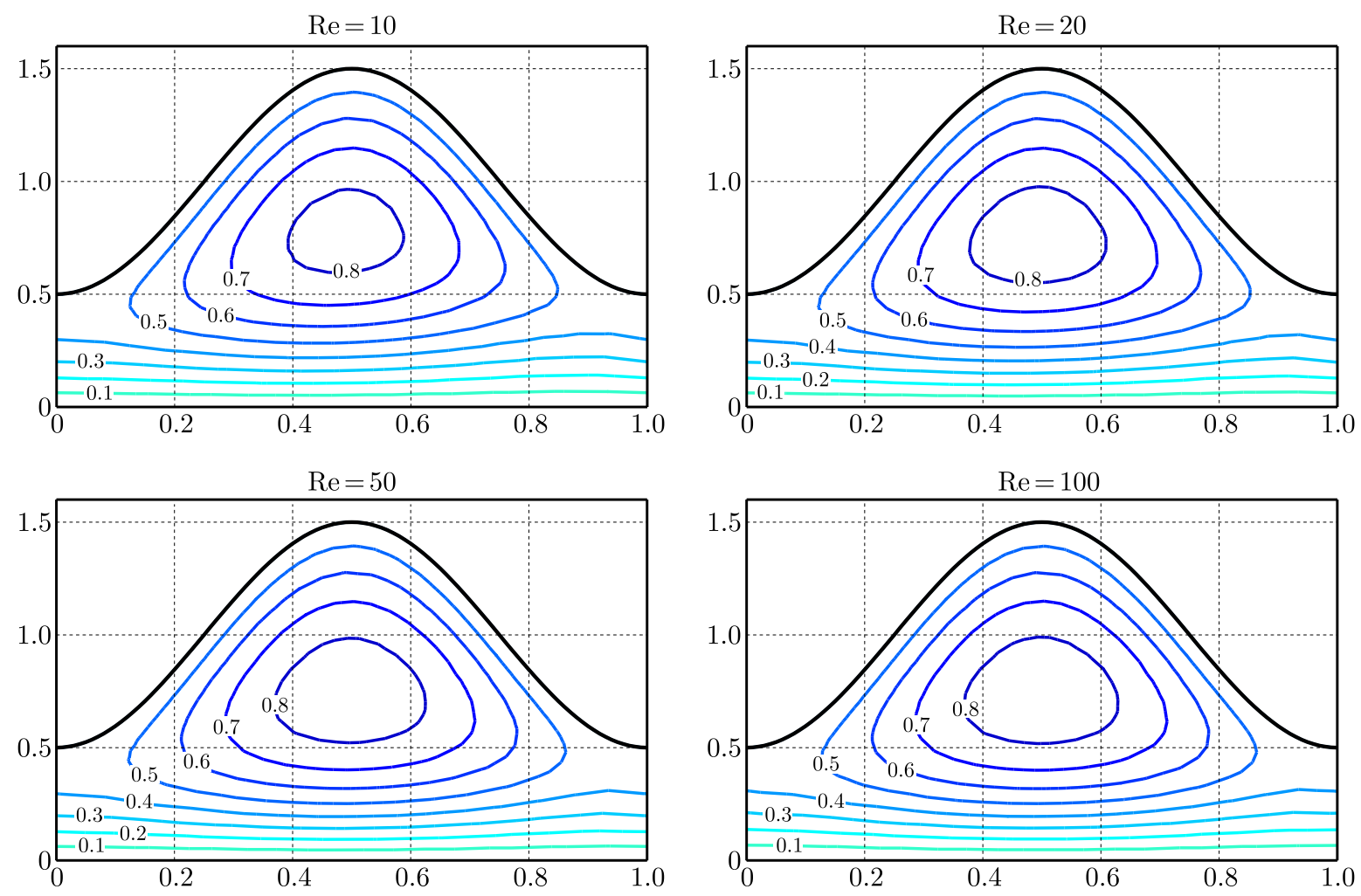

Fig. 9. Variation of stream lines in wave frame for different values of Re with $Q=1.4, \alpha=\phi=0.5$ 

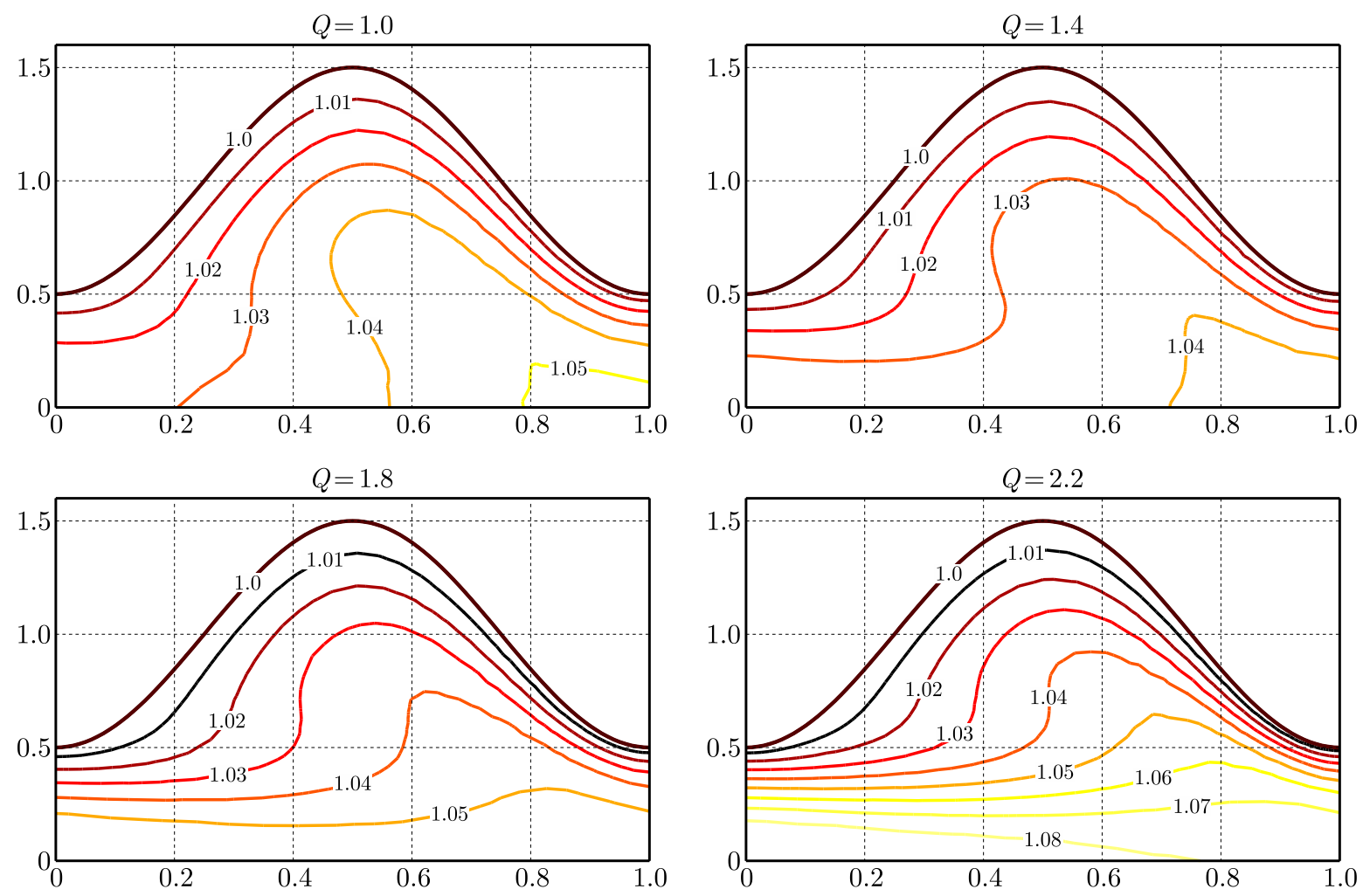

Fig. 10. Variation of isothermal lines in wave frame for different values of $Q$ with $\operatorname{Pr}=0.7, \operatorname{Re}=10$, $\alpha=\phi=0.5$ and $\beta=0.7$
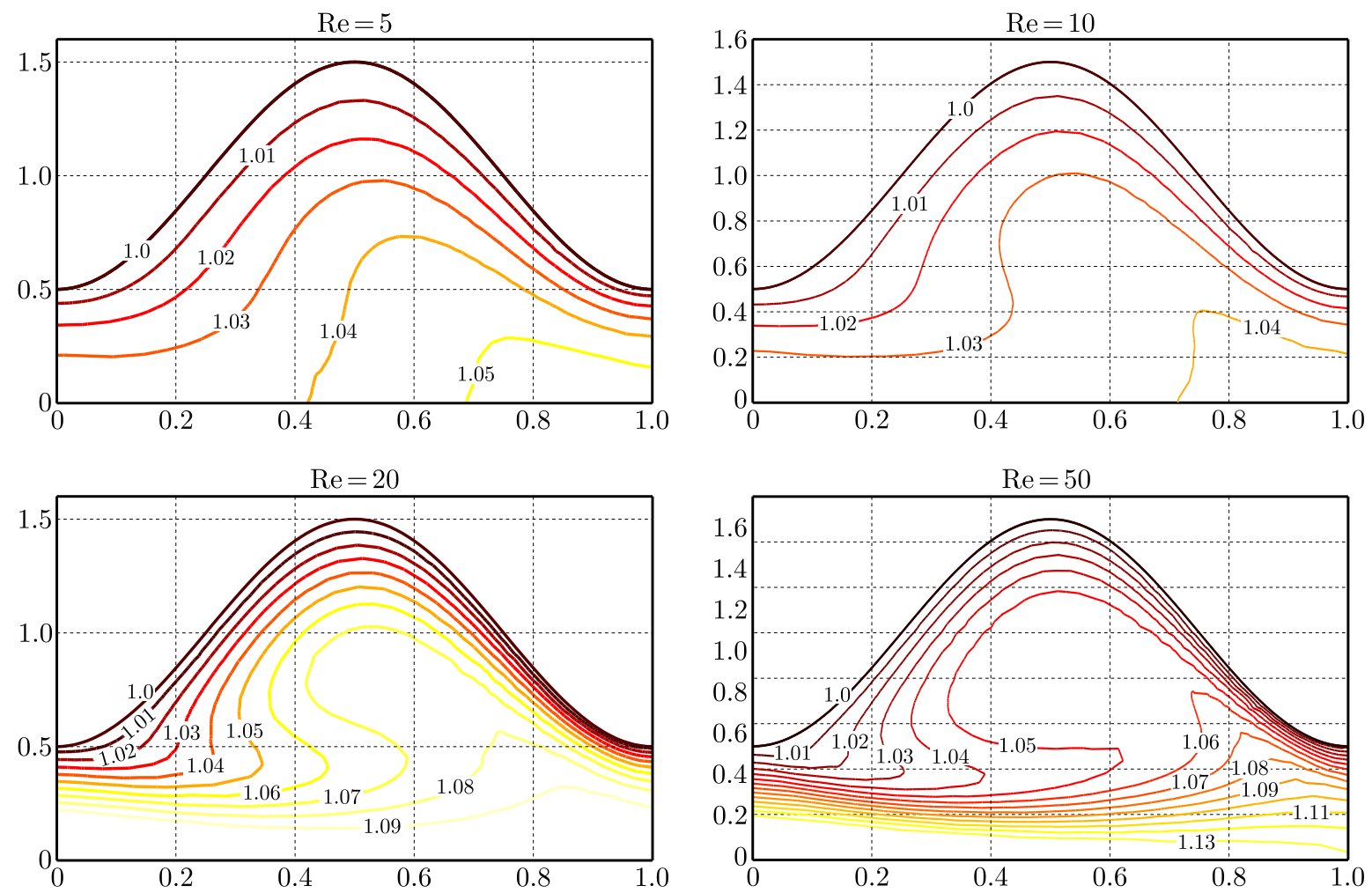

Fig. 11. Variation of isothermal lines in wave frame for different values of Re with $\operatorname{Pr}=0.7, Q=1.4$, $\alpha=\phi=0.5$ and $\beta=0.7$ 

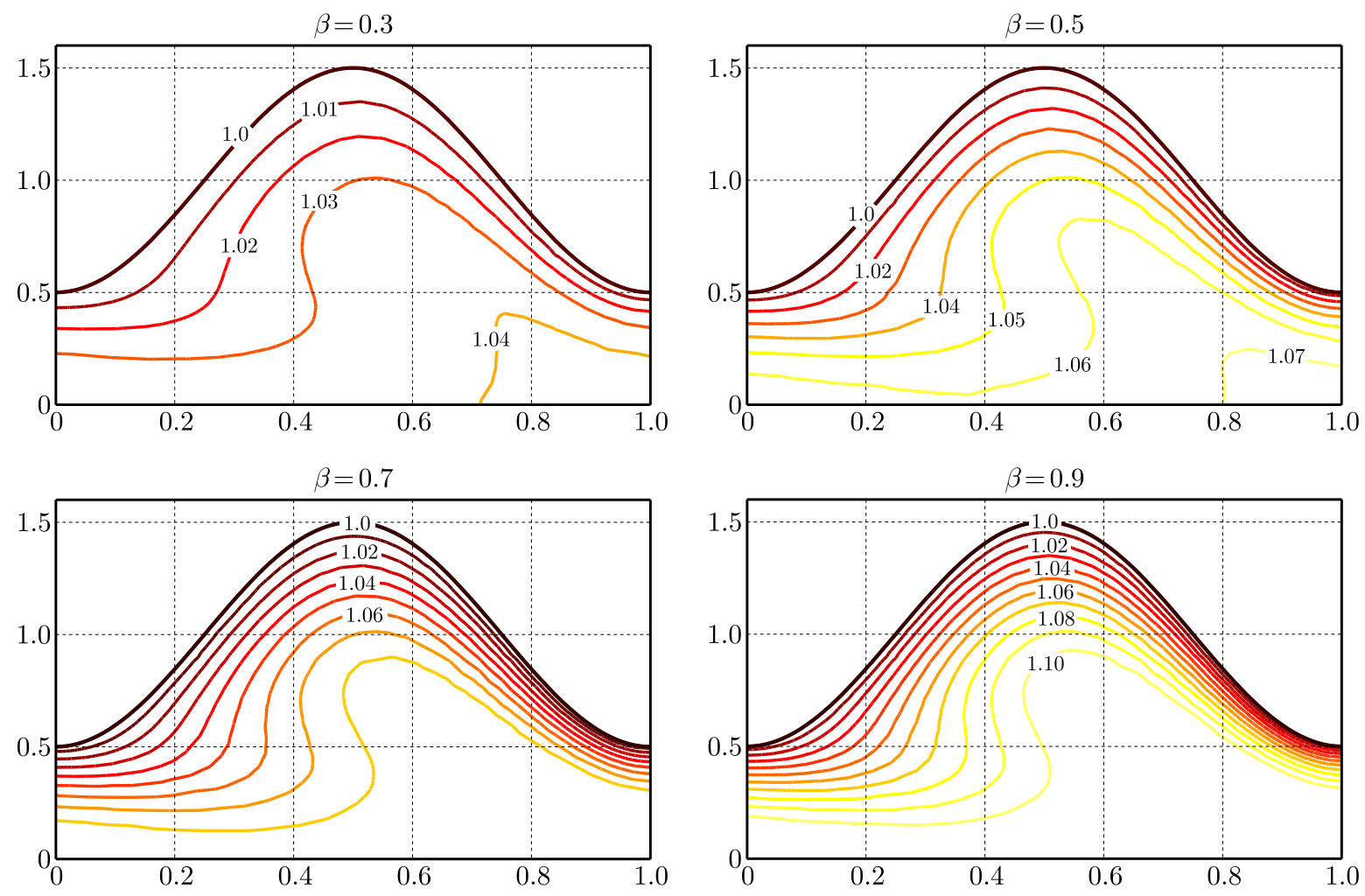

Fig. 12. Variation of isothermal lines for different values of $\beta$ with $\operatorname{Re}=10, Q=1.4, \alpha=\phi=0.5$ and $\operatorname{Pr}=0.7$
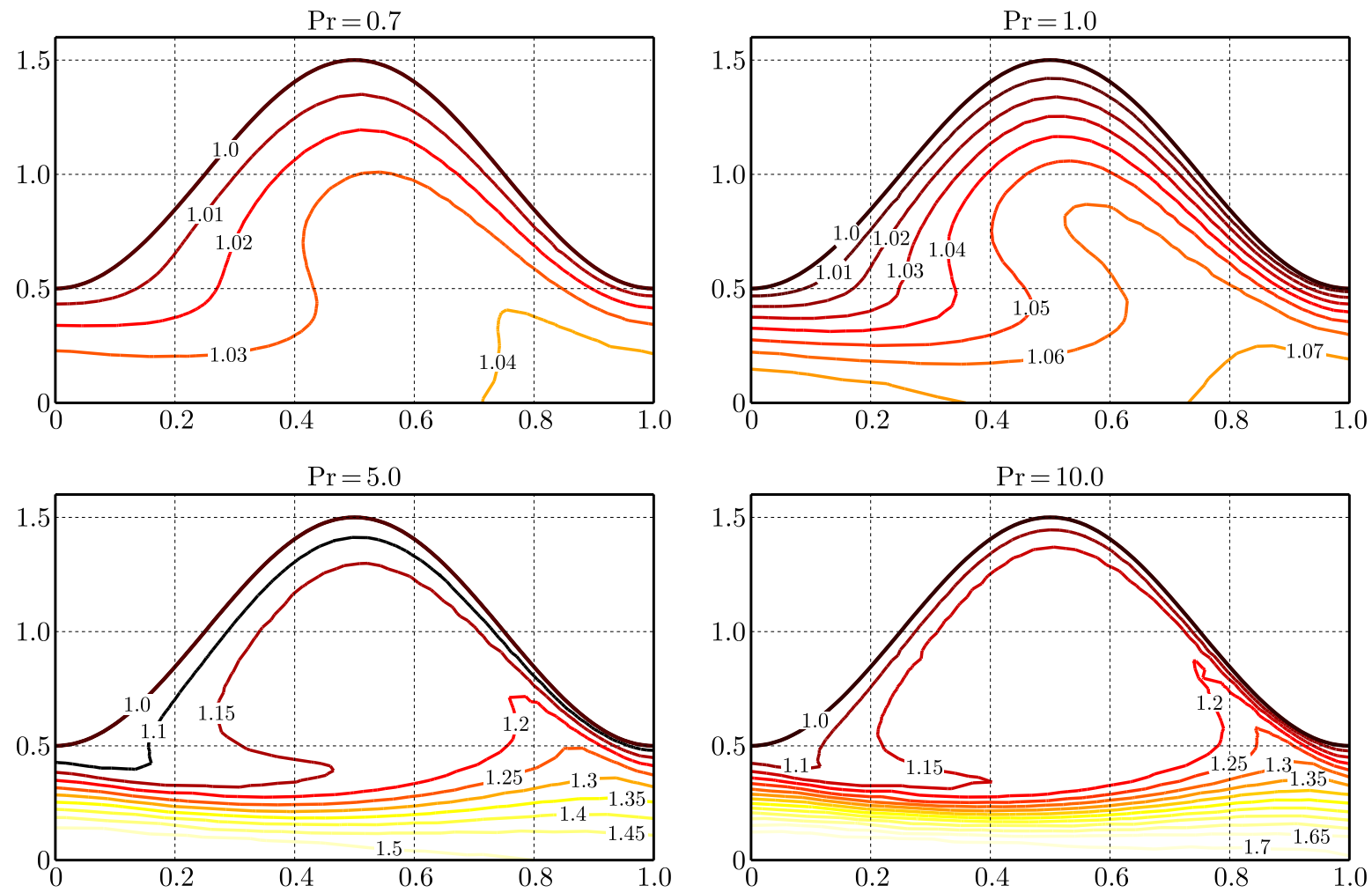

Fig. 13. Variation of isothermal lines for different values of $\operatorname{Pr}$ with $\operatorname{Re}=10, Q=1.4, \alpha=\phi=0.5$ and $\beta=0.7$ 


\subsection{Pressure field}

In this Section, we discussed the pressure rise per wave length against the time mean flow rate $Q$ in the fixed frame for different Reynolds and wave numbers. Figure 14a shows the graph of the pressure rise per wave length for different values of Re. We observe that the pressure graph is linear for small Reynolds numbers, and a nonzero curvature is noted for large Reynolds numbers. Also it is observed that the pressure increases with an increase in the Reynolds number when the time mean flow rate $Q \leqslant 0.45$ and decreases when $Q>0.45$. In Fig. 14b, the same behavior is observed in the case of wave numbers but the increasing region is less as compared with the Reynolds number, i.e. the pressure rise increases when $Q \leqslant 0.375$ and decreases $Q>0.375$. Also the alteration in pressure is much faster in the case of the Reynolds number as compared with that of the wave number when the time mean flow becomes zero.

(a)

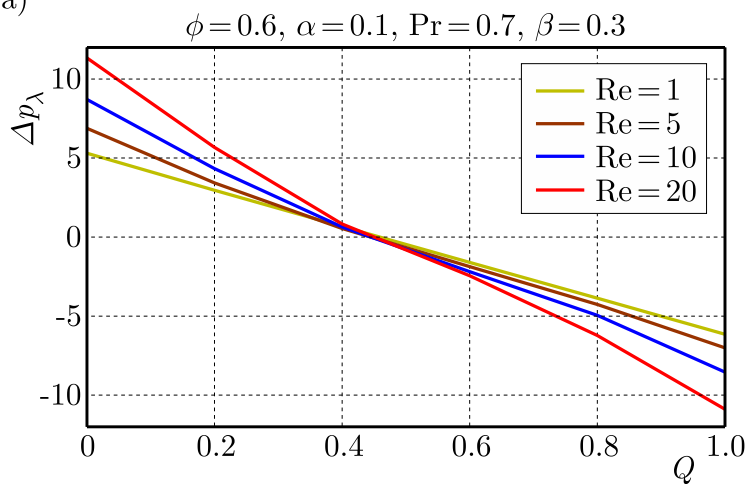

(b)

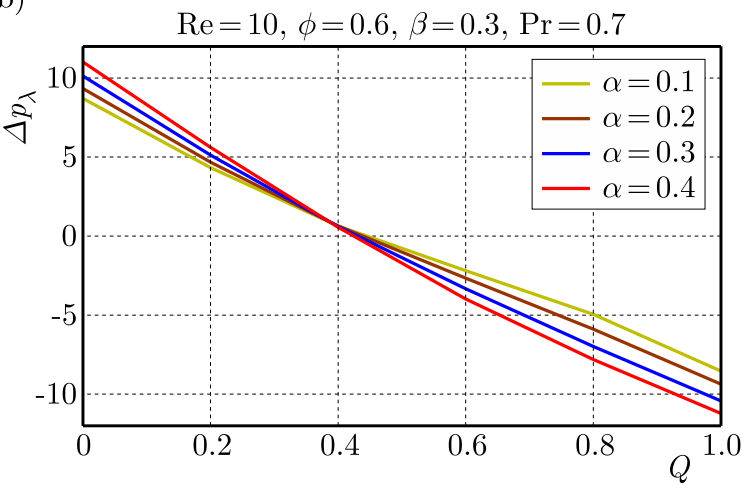

Fig. 14. Pressure rise per wave length for various values: (a) of Re against $\alpha=0.1$ and $\phi=0.5$, (b) of $\alpha$ against $\operatorname{Re}=10$ and $\phi=0.6$

\section{Conclusion}

The finite element analysis of the two-dimensional peristaltic flow with heat transfer is carried out by imposing any assumptions like in the earlier studies. The numerical result for the stream function, isothermal lines, pressure rise per wavelength, velocity and temperature profiles are shown graphically with different values of the parameter involved in the governing equations. The obtained results are also compared with the available results of Jaffrin (1973), which is valid only for low Reynolds numbers and for small wavelength. It is found that the obtained results are significantly different from the previous results without imposing any assumptions. The observations through the present study are summarized in the following points:

- The longitudinal velocity decreases near the wall of the channel by increasing the Reynolds number, but increases by increasing the wavelength.

- Temperature increases sharply by an increase in all the parameters while an increase in the wavelength reduces the temperature.

- The results are well convergent for large values of the Reynolds number.

- The circular trapped bolus increases with increasing values of the Reynolds number.

- The number of isothermal lines decreases near the wall by increasing the Prandtl number, and opposite behavior is observed for all other parameters.

- The pressure rise per wavelength increases in the augmented pumping region with the increasing Reynolds number and wavelength of the channel. 


\section{References}

1. Abo-Eldahab E., Barakat E., Nowar Kh., 2012, Hall currents and heat transfer effects on peristaltic transport in a vertical asymmetric channel through a porous medium, Mathematical Problems in Engineering, 1-23

2. Ali N., Hussain Q., Hayat T., Asghar S., 2008, Slip effects on the peristaltic transport of MHD fluid with variable viscosity, Physics Letters A, 372, 1477-1489

3. Eldabe N.T.M., El-Sayed M.F., Ghaly A.Y., Sayed H.M., 2008, Mixed convective heat and mass transfer in a non-Newtonian fluid at a peristaltic surface with temperature-dependent viscosity, Archive of Applied Mechanics, 78, 599-624

4. El-Sayed M.F., Eldabe N.T.M., Ghaly A.Y., Sayed H.M., 2011, Effects of chemical reaction, heat and mass transfer on non-Newtonian fluid flow through porous medium in a vertical peristaltic tube, Transport in Porous Media, 89, 185-212

5. Fung Y.C., Yıн C.S., 1968, Peristaltic transport, Transactions of the ASME, Journal of Applied Mechanics, 35, 669

6. Hayat T., Hina S., Ali N., 2010, Simultaneous effects of slip and heat transfer on the peristaltic flow, Communications in Nonlinear Science and Numerical Simulation, 15, 1526-1537

7. Hayat T., Hussain Q., Ali N., 2008, Influence of partial slip on the peristaltic flow in a porous medium, Physica A: Statistical Mechanics and its Applications, 387, 3399-3409

8. Hayat T., Mahomed F.M., Asghar S., 2005, Peristaltic flow of a magnetohydrodynamic Johnson-Segalman fluid, Non-Linear Dynamics, 40, 375-385

9. JAFFRIN M.Y., 1973, Inertia and streamline curvature effects on peristalsis, International Journal of Engineering Science, 11, 681-699

10. Kothandapani M., Srinivas S., 2008, On the influence of wall properties in the MHD peristaltic transport with heat transfer and porous medium, Physics Letters A, 372, 4586-4591

11. Kumar B.V.R., NAidu K.B., 1995, A numerical study of peristaltic flows, Computers and Fluids, 24, 161-176

12. Latham T.W., 1966, Fluid Motion in a Peristaltic Pump, Master thesis, MIT, Massachusetts

13. Lew H.S., Fung Y.C., Lowenstein C.B., 1971, Peristaltic carrying and mixing of chyme in the small intestine, Journal of Biomechanics, 4, 297-315

14. Mekheimer Kh.S., 2008, Peristaltic flow of a magneto-micropolar fluid. Effect of induced magnetic field, International Journal of Applied Mathematics, ID: 570825

15. Shapiro A.H., Jaffrin M.Y., Weinberg S.L., 1969, Peristaltic pumping with long wavelength at low Reynolds numbers, Journal of Fluid Mechanics, 37, 799

16. Srinivas S., Kothandapani M., , 2008, Peristaltic transport in an asymmetric channel with heat transfer - A note, International Communications in Heat and Mass Transfer, 35, 514-522

17. Takabatake S., Ayukawa K., 1982, Numerical study of two dimensional peristaltic flows, Journal of Fluid Mechanics, 122, 439-465

18. Takabatake S., Ayukawa K., Mori A., 1988, Peristaltic pumping in circular tubes: a numerical study of fluid transport and its efficiency, Journal of Fluid Mechanics, 193, 267-283

19. Takabatake S., Ayukawa K., Sawa M., 1987, Finite element analysis of two dimensional peristaltic flow (1st report, Finite element solution), Japan Society of Mechanical Engineers, 53, 12071213

20. Takabatake S., Ayukawa K., Sawa M., 1990, Finite element analysis of two dimensional peristaltic flow (2nd report, Pessure-flow characteristics), Japan Society of Mechanical Engineers, 56, 3633-3637 
21. Tripathi D., 2013, Study of transient peristaltic heat flow through a finite porous channel, Mathematical and Computer Modelling: An International Journal, 57, 5, 1270-1283

22. Weinberg S.L., Eckstein E.C., Shapiro A.H., 1971, An experimental study of peristaltic pumping, Journal of Fluid Mechanics, 49, 461

23. Yi M., BAu H.H., Hu H., 2002, Peristaltically induced motion in a closed cavity with two vibrating walls, Physics of Fluids, 14, 184-197

Manuscript received November 20, 2018; accepted for print February 11, 2021 